\title{
2 Effects of Bacterial Inactivation Methods on Downstream Proteomic Analysis
}

3 Andy Lin $^{1}$, Eric D. Merkley ${ }^{1}$, Brian H. Clowers ${ }^{1,2}$, Janine R. Hutchison ${ }^{1}$, and Helen W.

$4 \operatorname{Kreuzer}^{1} *$

5

$6 \quad{ }^{1}$ Signatures Sciences \& Technology Division, National Security Directorate, Pacific Northwest

$7 \quad$ National Laboratory, Richland, WA 99352

$8 \quad{ }^{2}$ Department of Chemistry, Washington State University, Pullman, WA 99164

9

10 Correspondence:

11 Helen W. Kreuzer

12 Pacific Northwest National Laboratory

13902 Battelle Boulevard,

14 P.O. Box 999, MSIN: P7-50

15 Richland, WA 99352

16 Email: Helen.Kreuzer@pnnl.gov

17 Phone: 1-509-371-6799

18 Fax: 1-509-375-2227

19

20

21 
22 Abstract:

Inactivation of pathogenic microbial samples is often necessary for the protection of

24 researchers and to comply with local and federal regulations. By its nature, biological

25 inactivation causes changes to microbial samples, potentially affecting observed experimental

26 results. While inactivation-induced damage to materials such as DNA has been evaluated, the

27 effect of various inactivation strategies on proteomic data, to our knowledge, has not been

28 discussed. To this end, we inactivated samples of Yersinia pestis and Escherichia coli by

29 autoclave, ethanol, or irradiation treatment to determine how inactivation changes liquid

30 chromatography-tandem mass spectrometry data quality as well as apparent protein content of

31 cells. Proteomic datasets obtained from aliquots of samples inactivated by different methods

32 were highly similar, with Pearson correlation coefficients ranging from 0.822 to 0.985 and 0.816

33 to 0.985 for $E$. coli and $Y$. pestis, respectively, suggesting that inactivation had only slight

34 impacts on the set of proteins identified. In addition, spectral quality metrics such as distributions

35 of various database search algorithm scores remained constant across inactivation methods,

36 indicating that inactivation does not appreciably degrade spectral quality. Though overall

37 changes resulting from inactivation were small, there were detectable trends. For example, one-

38 sided Fischer exact tests determined that periplasmic proteins decrease in observed abundance

39 after sample inactivation by autoclaving $\left(\alpha=1.71 \times 10^{-2}\right.$ for E. coli, $\alpha=4.97 \times 10^{-4}$ for Y. pestis $)$

40 and irradiation $\left(\alpha=9.43 \times 10^{-7}\right.$ for E. coli, $\alpha=1.21 \times 10^{-5}$ for $Y$. pestis $)$ when compared to

41 controls that were not inactivated. Based on our data, if sample inactivation is necessary, we

42 recommend inactivation with ethanol treatment with secondary preference given to irradiation.

43 Keywords: 
44 liquid chromatography tandem mass spectrometry; microbial inactivation; proteomics;

45 Enterobacteriaceae

46 1. Introduction

Mass spectrometry-based proteomics has become an increasingly important tool for 48 examining changes in protein content of microorganisms under varied experimental conditions.

49 However, many research groups do not have the necessary equipment and experience to perform 50 proteomic analysis by mass spectrometry; therefore samples generated by one research group 51 have to be shipped to a second group with proteomic capability. Regulations surrounding

52 shipping and handling of microorganisms are dictated by the biological risk associated with each 53 organism. Microorganisms are assigned biosafety levels (BSL) that range from 1-4 (Centers for 54 Disease Control and Prevention, 2009; Zaki, 2010). The BSL assigned to each microorganism 55 specifies the handling precautions and shipping requirements for a particular microorganism. For 56 example, BSL-1 organisms such as non-pathogenic Escherichia coli can be handled on an open 57 laboratory bench, as well as transferred to other facilities with minimal preparation. Organisms 58 with a BSL rating of 2 or higher must be inactivated (non-viable and non-infectious state) before 59 they can be removed from the laboratory. Sample inactivation also occurs in scenarios outside of 60 research laboratories. Since the 2001 anthrax letter cases (in which letters containing live $B$.

61 anthracis were sent through the U.S. mail), mail sent to Congress, the White House, and other 62 federal agencies is irradiated to kill potential biological agents (Government Accountability 63 Office, 2008). As a result, samples that have been sent through the U.S. mail system, perhaps 64 recovered in a forensic investigation, will have been inactivated by irradiation.

65 The effectiveness of irradiation and other procedures for inactivating microorganisms has 66 been widely documented (Coohill and Sagripanti, 2008; Lasch et al., 2008; Rose and O'Connell, 
67

2009; Vatansever et al., 2013; Waje et al., 2009; Whitney et al., 2003). In addition, several studies have assessed the effects of inactivation on microbial identification by ELISA and PCR (Sagripanti et al., 2011; Talbot et al., 2010), and MALDI-TOF MS (Lasch et al., 2008; Talbot et al., 2010). However, the effects of various inactivation methods on liquid chromatography tandem mass spectrometry (LC-MS/MS) data quality and apparent protein expression profiles of cells has to our knowledge not been investigated. To this end we evaluated the effects of irradiation, ethanol treatment, and autoclaving, on observed expression profiles in Escherichia coli and Yersinia pestis. Our results demonstrate that the inactivation procedures we tested have minimal impact on apparent protein expression and spectral data quality.

\section{Methods}

\subsection{Bacterial strains and culture conditions}

Bacterial strains used for this research were E. coli ATCC 15597 (obtained from the American Type Culture Collection) and Y. pestis KIM D27, an isogenic derivative of KIM 10+ (biovar Mediaevalis) that was passaged until the pigmentation phenotype was lost due to spontaneous deletion of the pgm locus (Une and Brubaker, 1984). We obtained Y. pestis KIM D27 from Ellen Panisko (Pacific Northwest National Laboratory), who received it from Vladimir Motin at the University of Texas Medical Branch. Both E. coli ATCC 15597 and Y. pestis pgmcultivation and manipulation were done at BSL-2 conditions until bacterial inactivation was verified.

All E. coli ATCC 15597 cultures were cultured in TSB broth or agar (TSB; BD 286220) at $37^{\circ} \mathrm{C}$. Y. pestis KIMD27 was cultured in Brain Heart Infusion broth or agar (BHI; BD $211059)$ at $30^{\circ} \mathrm{C}$. Starter cultures were established by streaking frozen glycerol stocks onto the appropriate agar plate and were incubated overnight at the appropriate temperature. To achieve 
90 biological replication, three replicate broth cultures were inoculated with separate individual

91 colonies in $50 \mathrm{~mL}$ of the appropriate broth medium in $250 \mathrm{~mL}$ glass flasks. These cultures were

92 incubated for 16 hours at the appropriate temperature with shaking at $150 \mathrm{rpm}$. Bacteria were

93 collected by centrifugation for 10 minutes at $5500 \mathrm{x} g$ in a Thermo-Fisher swinging bucket

94 centrifuge. The supernatant was decanted and the pellet was washed twice in $50 \mathrm{~mL}$ of

95 phosphate-buffered saline (PBS; Gibco 20012). The washed pellet was brought to a final volume

96 of $50 \mathrm{~mL}$ in PBS. Bacteria were enumerated by plating on the appropriate media prior to

97 inactivation.

\section{$98 \quad 2.2$ Sample Inactivation}

99 In addition to the untreated control, three inactivation treatments were used on $12 \mathrm{~mL}$ of

100 each culture: irradiation, ethanol, and autoclaving. The control and irradiated samples were

101 placed into $15 \mathrm{~mL}$ conical tubes while the other samples were placed in $50 \mathrm{~mL}$ conical tubes.

102 Bacteria were irradiated with a Colbalt- 60 source for 24 hours at $0.47 \mathrm{kGy} / \mathrm{h}$ for a total exposure 103 of $11.3 \mathrm{kGy}$. Autoclaved samples were exposed for 20 minutes at $30 \mathrm{psi}$ [206.8 kPa] and $121{ }^{\circ} \mathrm{C}$ 104 in a Getinge autoclave. For the ethanol treatment, ethanol was added to the PBS cell suspension 105 for a final concentration of $40 \%$ ethanol, and the cells were incubated in this solution for 30 106 minutes at room temperature. Following ethanol inactivation treatments, cells were transferred to 107 a clean tube and were collected by centrifugation and resuspended in PBS, transferred to a new 108 tube, collected by centrifugation and brought up to a final volume of $12 \mathrm{~mL}$ PBS. Ten percent of 109 each sample was plated on the appropriate bacterial culture media and incubated for 48 hours to 110 verify that the cells had been inactivated and were non-viable. All samples were stored at $4{ }^{\circ} \mathrm{C}$ 111 prior to proteomic preparation.

\section{2.3 Whole Mount Transmission Electron Micrographs}


After inactivation, bacteria were fixed in $2.5 \%$ glutaraldehyde in PBS (Electron

114

Microscopy Sciences, Hatfield, PA, USA \# 16000) for transmission electron micrograph (TEM)

imaging. Cells were stained with Nano-W (Nanoprobes, Yaphank, NY, USA \#2018) prior to imaging on a Tecnai T-12 transmission electron microscope (FEI Co., Hillsboro, OR) operating at $120 \mathrm{kV}$ with a LaB filament. Images were collected digitally using a 2x2K UltraScan 1000 charge-coupled device (Gatan Inc., Pleasanton, CA).

\subsection{Digestion of Proteins}

For peptide preparation, $\sim 4 \times 10^{8} \mathrm{CFU} Y$. pestis or of $\sim 2 \times 10^{9} \mathrm{CFU}$ E. coli were pelleted and resuspended in $8 \mathrm{M}$ urea and $0.5 \mathrm{mM}$ 2-mercaptoethanol in Tris-HCl, $\mathrm{pH}$ 8. Samples were incubated for one hour at $60{ }^{\circ} \mathrm{C}$ with shaking at $300 \mathrm{rpm}$. Insoluble cell material was removed by centrifugation. To the supernatant, $400 \mu \mathrm{L}$ of $50 \mathrm{mM}$ ammonium bicarbonate was added followed by $1 \mu \mathrm{g}$ of trypsin (Promega; \# V5280). Samples were incubated at $37^{\circ} \mathrm{C}$ overnight. Solid phase extraction (SPE) was performed with a vacuum manifold using Strata C-18 T columns following the manufacturer's protocol. The flow rate for vacuuming steps was 0.5 $\mathrm{mL} / \mathrm{min}$; the vacuum seal was released after each solution was removed. Briefly, $1 \mathrm{~mL}$ of $100 \%$ methanol (Chromasolv, Sigma Aldrich, St. Louis, MO) was added to activate the resin, followed by a conditioning rinse of $1 \mathrm{~mL}$ of $0.1 \%$ trifluoroacetic acid (TFA, Sigma Aldrich, St. Louis, MO) water, followed by the sample. The samples were washed with $5 \%$ acetonitrile in $0.1 \%$ TFA water, and eluted with $80 \%$ acetonitrile in $0.1 \%$ TFA water into clean, low protein-binding $1.5 \mathrm{~mL}$ microfuge tubes. Samples were dried to near completeness $(5-10 \mu \mathrm{L}$ remaining) with a Thermo-Fisher SpeedVac and resuspended in $25 \mu \mathrm{L}$ of $0.5 \%$ formic acid. The resuspended samples were transferred to high performance liquid chromatography (HPLC) vials with $250 \mu \mathrm{L}$ inert glass inserts, capped with screw caps, and stored at $-20^{\circ} \mathrm{C}$ prior to analysis. 


\subsection{Liquid Chromatography Tandem Mass Spectrometry}

Reverse-phase liquid chromatography separation of digested peptide samples was

138 performed on an Agilent Infinity 1260 HPLC system, using a column consisting of a fused silica 139 capillary (40 cm long x $150 \mu \mathrm{m}$ inner diameter) packed in-house with Jupiter C18 resin $(5-\mu \mathrm{m}$ 140 particle size, $300 \AA$ A pore size; Phenomenex, Torrance, California). Mobile phases were: A, 5\% 141 acetonitrile/ $0.1 \%$ formic acid; and B, 95\% acetonitrile/ $0.1 \%$ formic acid. Peptides were eluted 142 during the following 160-min gradient: 100\% Solvent A for 10 min; 0\% B to 7.5\% B over 1 min; $1437.5 \%$ B to $45 \%$ B over $109 \mathrm{~min} ; 45 \%$ B to $95 \%$ B over $2 \mathrm{~min} ; 95 \%$ B for $10 \mathrm{~min}, 95 \%$ B to $0 \%$ 144 B over 4 min and 100\% A for 23 min. Column washes $(5-\mu \mathrm{L}$ injections of $50 \%$ isopropyl 145 alcohol $/ 50 \%$ acetone/ $0.1 \%$ formic acid) were run between sample injections. To achieve 146 analytical replication, each sample was injected three times. Run order was randomized to 147 prevent statistical artifacts relating to run order or column age.

The eluent from the liquid chromatography was introduced directly into a Thermo 149 Scientific LTQ Orbitrap XL mass spectrometer via electrospray ionization using an etched silica 150 capillary as the emitter (Kelly et al., 2006). Precursor (survey) mass spectra were acquired using 151 the Orbitrap mass analyzer at a resolution of 30,000, followed by collision-induced dissociation 152 (CID) fragment ion scans of the seven most abundant precursors, collected at low resolution 153 using the ion trap mass analyzer. CID normalized collision energy was $35 \%$ and monoisotopic 154 precursor selection was enabled. Dynamic exclusion of precursors already selected for 155 fragmentation was applied (repeat count 2, repeat duration $30 \mathrm{~s}$, exclusion list size 250, exclusion 156 list duration $180 \mathrm{~s})$.

$157 \quad 2.6$ Data Processing and Analysis 
LC-MS/MS data was analyzed to obtain peptide-spectrum matches (PSMs) using a three step pipeline. DeconMSn (Mayampurath et al., 2008) preprocessed the data by accurately determining the monoisoptic mass and the charge state of parent ions from tandem mass spectrometry. Additional preprocessing was done with DTARefinery (Fenyö and Beavis, 2003; Petyuk et al., 2010), which uses a high-stringency X!Tandem search to recalibrate precursors masses for correction of systematic mass drift. Afterward the post-processed data was searched against a database using MS-GF+ (Kim et al., 2008). E. coli data was searched against the K12 strain proteome and $Y$. pestis data was searched against the KIM D10+ strain proteome, which were both downloaded from PATRIC (Wattam et al., 2014). Both proteome databases were concatenated with a contaminant database, which includes common contaminants such as human keratin, trypsin, and bovine albumin. Options used in the MS-GF+ search included using semitryptic peptide ends, a parent mass tolerance of 20 parts per million (ppm), and an optional methionine oxidation modification. Minimum and maximum lengths of the peptide were 0 and 50 amino acids, respectively, and the isotope error range was between -1 and 3 . Additional options include reporting q-values and decoy PSMs as well as reporting PSMs that match to multiple proteins on a single line instead of multiple lines (Kim et al., 2008). The target-decoy approach was used to create an estimation of the false discovery rate (FDR) using reverse protein sequences as decoys (Benjamini and Hochberg, 1995; Elias and Gygi, 2007).

Data resulting from individual LC-MS/MS runs were merged by organism and treatment type. In general, search results from nine LC-MS/MS runs were merged (three technical replicates of each of three biological replicates). Due to sample limitations only seven LCMS/MS runs for the ethanol-inactivated $Y$. pestis sample were completed. Peptides were filtered to a $1 \%$ FDR using the q-value and by removing any peptides that had a precursor mass error 
greater than 2 or less than $-2 \mathrm{ppm}$. Additionally, to consider a protein confidently identified, at

182 least two different peptides from that protein had to be detected in each individual dataset.

183 Proteins were quantified using spectral counts (Liu et al., 2004). For each organism, samples

184 inactivated by each of the three methods were compared against an untreated control, resulting in

185 six comparisons. Significance testing between inactivated samples and the control samples was

186 conducted using Quasitel at a protein FDR of 5\% (Li et al., 2010). This software, which uses a

187 quasi-Poisson likelihood model for determining statistical significance of protein expression

188 changes measured by spectral count data, performs well for high spectral counts, but

189 overestimates significance when spectral counts are low (fewer than three to five spectral

190 counts). Therefore, an additional filter removed proteins considered significantly changing that

191 had zero spectral counts in one condition and very few spectral counts in the second condition.

192 This heuristic requires the average number of spectral counts over all runs for a protein in a

193 specific condition to be at least three when the other condition has zero spectral counts. Initial

194 gene ontology enrichment categories was explored using the Database for Annotation,

195 Visualization and Integrated Discovery (Huang et al., 2008, 2009). Subcellular localizations of

196 proteins was determined using version 3 of PSORTb (Yu et al., 2010). Manual annotation was

197 conducted using Uniprot for proteins for which PSORTb was unable to determine the subcellular

198 localization of (Consortium, 2014).

199 3. Results

$200 \quad 3.1$ Inactivation of bacterial cultures coli and $Y$. pestis cultures, as measured by lack of growth on bacterial culture media after 
208

treatment. Inactivated cells were

examined by electron microscopy to

assess any morphological changes

associated with each treatment. To

discover potential effects of each

inactivation method on proteomic data,

the identified proteomes of the inactivated

samples were compared to those of the

untreated control samples.

\subsection{Transmission Electron Microscopy:}

TEM was used to visualize control
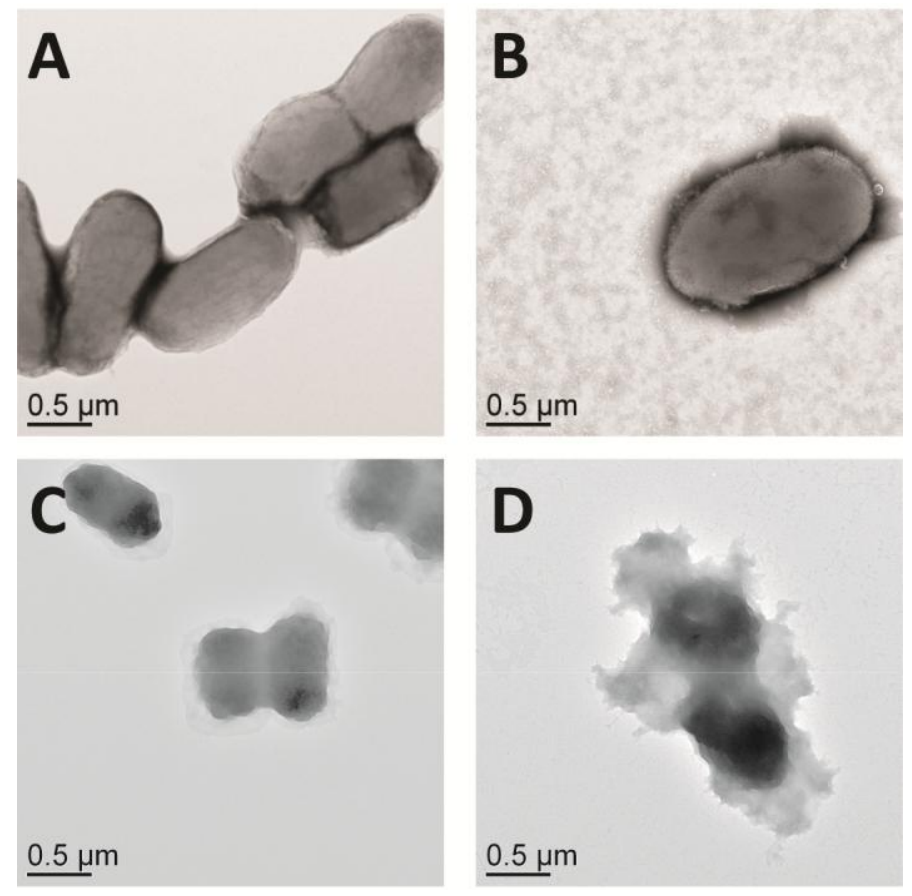

Figure 1: Whole mount transmission electron micrographs of control and inactivated $Y$. pestis sample: (A) no treatment; (B) irradiated; (C) ethanol; (D) autoclaved.

and inactivated $Y$. pestis and E. coli samples in order observe changes in cell morphology resulting from inactivation (Figure 1, Figure S1). Clearly defined membranes can be seen in the

$Y$. pestis control samples, which maintained the classical morphology of a rod-shaped

coccobacillus (Figure 1A). Irradiated cells were shorter and rounder compared to the control cells. Irradiated and ethanol-treated samples display membranes that appear detached from the core of the cell (Figure 1B, 1C). The autoclaved cells showed the greatest morphological change, with the membrane disrupted and dissociated from the cell core (Figure 1D). Ethanol-treated and autoclaved cells were also agglomerated and appeared to fuse with neighboring cells. Overall, the morphology of the autoclaved cells is most dissimilar to the control sample while irradiated cells are most similar to the control sample.

\subsection{Proteomic Analysis}

\subsubsection{Data Quality Is Unaffected by Inactivation}


Prior to determining what effect inactivation had on the observed abundances of specific protein we assessed what influence inactivation had on spectral quality, if any. Four metrics were used to evaluate whether an inactivation method impacted data quality: (1) the number of unique peptides and proteins found in inactivated samples and controls, (2) the distribution of peptidespectrum matches (PSMs) per protein, (3) histograms of filtered and unfiltered raw MSGF+ scores, and (4) histograms of MS-GF+ DeNovoScores. Based upon these measures, proteomic data quality is largely unaffected by the inactivation methods examined.

The first metric compared the number of unique peptides and proteins found in a particular inactivation condition to the control. The more unique peptides and proteins found in a particular condition and not in the control and vice versa, the larger the difference. In general, the total number of unique and shared peptides and proteins were similar across a given strain for the three treatments (Table 1, Figure S2). This comparison indicates that data quality is unaffected since the same peptides and proteins are, in general, being identified. Interestingly, it appears that in the autoclaved E. coli samples the proportion of peptides and proteins unique to the control are

\begin{tabular}{|c|c|c|c|c|c|c|c|}
\hline \multirow[b]{2}{*}{ Strain } & \multirow[b]{2}{*}{ Treatment } & \multicolumn{3}{|c|}{ Peptides detected } & \multicolumn{3}{|c|}{ Proteins detected } \\
\hline & & $\begin{array}{l}\text { Unique to } \\
\text { treatment }\end{array}$ & $\begin{array}{c}\text { Detected in } \\
\text { both }\end{array}$ & $\begin{array}{c}\text { Unique to } \\
\text { Control }\end{array}$ & $\begin{array}{l}\text { Unique to } \\
\text { treatment }\end{array}$ & $\begin{array}{c}\text { Dected in } \\
\text { both }\end{array}$ & $\begin{array}{c}\text { Unique to } \\
\text { Control }\end{array}$ \\
\hline \multirow{6}{*}{ E. coli } & \multirow{2}{*}{ Autoclave } & 969 & 2947 & 1834 & 92 & 595 & 243 \\
\hline & & $16.9 \%$ & $52.3 \%$ & $31.9 \%$ & $9.9 \%$ & $64.0 \%$ & $26.1 \%$ \\
\hline & \multirow{2}{*}{ Ethanol } & 1070 & 3729 & 1052 & 125 & 717 & 121 \\
\hline & & $18.3 \%$ & $63.7 \%$ & $18.0 \%$ & $13.0 \%$ & $74.5 \%$ & $12.6 \%$ \\
\hline & \multirow{2}{*}{ Irradiation } & 1015 & 3828 & 953 & 79 & 699 & 139 \\
\hline & & $17.6 \%$ & $66.3 \%$ & $16.2 \%$ & $8.6 \%$ & $76.2 \%$ & $15.2 \%$ \\
\hline \multirow{6}{*}{$Y$. pestis } & \multirow{2}{*}{ Autoclave } & 1303 & 3039 & 1921 & 129 & 634 & 155 \\
\hline & & $20.8 \%$ & $48.5 \%$ & $30.7 \%$ & $14.1 \%$ & $69.3 \%$ & $16.9 \%$ \\
\hline & \multirow{2}{*}{ Ethanol } & 1839 & 3411 & 1539 & 178 & 673 & 116 \\
\hline & & $27.1 \%$ & $50.0 \%$ & $22.7 \%$ & $18.4 \%$ & $69.6 \%$ & $12.0 \%$ \\
\hline & \multirow{2}{*}{ Irradiation } & 1865 & 3520 & 1440 & 222 & 668 & 121 \\
\hline & & $27.3 \%$ & $51.6 \%$ & $12.1 \%$ & $22.0 \%$ & $66.1 \%$ & $12.0 \%$ \\
\hline
\end{tabular}

Table 1: Table comparing the number of distinct peptides and proteins that are found in treated E. coli and Y. pestis sample when compared to the control samples. As an example, in E. coli there are 969 distinct, $\sim 16.9 \%$, peptides that are identified in the autoclaved samples and not the control samples. Data from all biological and technical replicates are combined. 
larger than found for the other two inactivation methods. A similar trend was observed in the $Y$.

242 pestis autoclaved samples. This suggests that autoclaving may change the observed proteome

243 more than irradiation or ethanol treatment. The other three metrics support the conclusion that the overall quality of proteomic data is not degraded by inactivation. The distribution of PSMs per protein is about the same for all inactivation methods for both organisms (Figure S3). A histogram of the filtered and unfiltered MSGFScores, which is a raw score, is also about the same for all inactivation methods for both organisms (Figure S4 and S5). In the unfiltered histograms, the presence of a $2^{\text {nd }}$ mode centered on a MSGF score of $\sim 100$ indicates that there are a large number of high scoring hits (Figure S4 and S5). The distributions of DeNovoScores, across all inactivation methods, are of the same size and shape (Figure S6 and S7). The DeNovoScore is a raw score representing the best-scoring peptide in theoretical sequence space, regardless of whether it is seen in the database; thus, it serves as a measure of spectral quality. These distribution shows that there are a large number of high-quality spectra in the LC-MS/MS runs and that spectrum quality was not degraded as a result any inactivation method.

\subsubsection{Small Changes in Apparent Protein Abundance Occur after Inactivation}

After determining that inactivation did not negatively impact data quality, we sought to discover changes in observed protein abundance induced by inactivation. Any changes to the observed proteome as a result of inactivation were minor. Figure 2 presents heat maps of the Pearson correlation coefficient of protein abundances between each pair of datasets (LC-MS/MS runs) for both organisms. The Pearson correlation coefficient between any two LC-MS/MS runs ranged from 0.822 to 0.985 and 0.816 to 0.985 for E. coli and $Y$. pestis, respectively (Figure 2). In addition, the weakest Pearson correlation coefficient between any two individual data sets from different inactivation conditions is 0.822 for $E$. coli and 0.815 for $Y$. pestis, compared to 

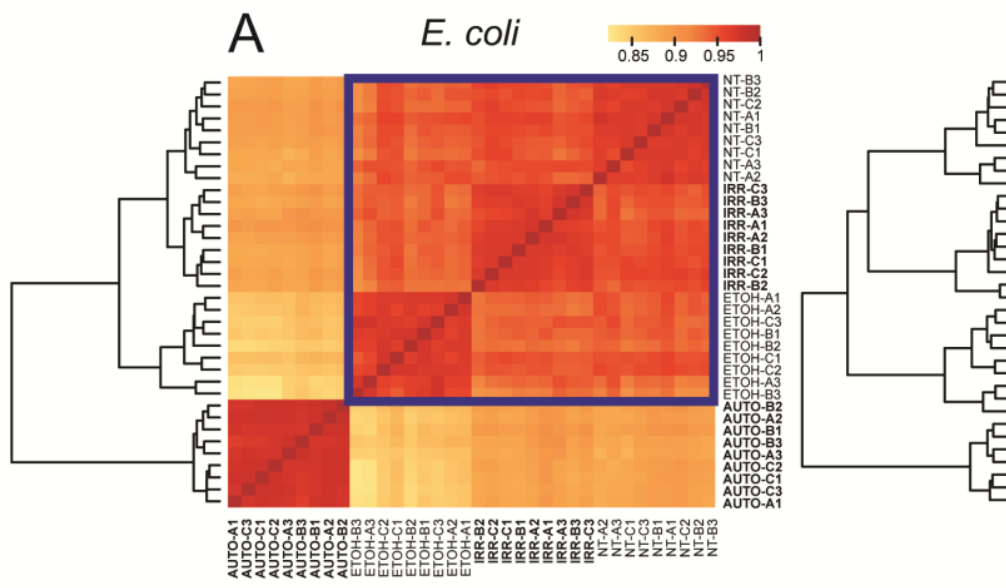

Figure 2: Heat map of the Pearson correlation coefficient between each dataset (LC-MS/MS run). The dark red colors indicate a greater correlation coefficient while a yellow and orange color represents a lower correlation coefficient. A, B, and C represent different sample replicates while the numbers 1,2 , and 3 represent technical replicates.

0.945 and 0.955 for E. coli and Y. pestis in the same condition (Figure 2). Therefore, for the vast majority of the proteome, observed protein abundances remain stable regardless of inactivation method.

Though the proteomes observed for all samples of E. coli and Y. pestis are similar, the proteomes of samples inactivated by each method could be distinguished from samples inactivated by different methods. In Figure 2 this discrimination is displayed by the fact that samples inactivated by the same method cluster with each other and do not cluster with samples inactivated by different methods, suggesting that each inactivation method imparts reproducible changes on the proteins in the sample and thus, the identified proteome.

For $Y$. pestis, the samples from each inactivation method, as well as the control samples, correlated most highly with samples that underwent the same treatment, resulting in blocks of dark red. For example, the dark red block in the upper right corner indicates that the expression patterns of the control samples are more similar to one another than to those of inactivated samples. This trend also occurs in E. coli, though it is not as distinct. In E. coli the non-treated, irradiated, and ethanol treated samples are more similar to each other (Figure 2, blue outline) 
7280 (Figure 2, lower left hand corner). At the

same time the protein profiles from the control, irradiated, and ethanol treatment

283 samples in E. coli are all more highly

284 correlated with each other than with any

285 other treatment type, resulting in the 3

286 smaller red boxes within the blue outline.

287 The differentiation between

288 inactivation methods can also be

289 visualized by a principal component

290 analysis of the protein abundances of

291 each LC-MS/MS run in E. coli and $Y$.

292 pestis (Figure 3). Samples inactivated by

293 the same method appear with each other

294 while samples that are inactivated by

295 unlike methods do not appear together.
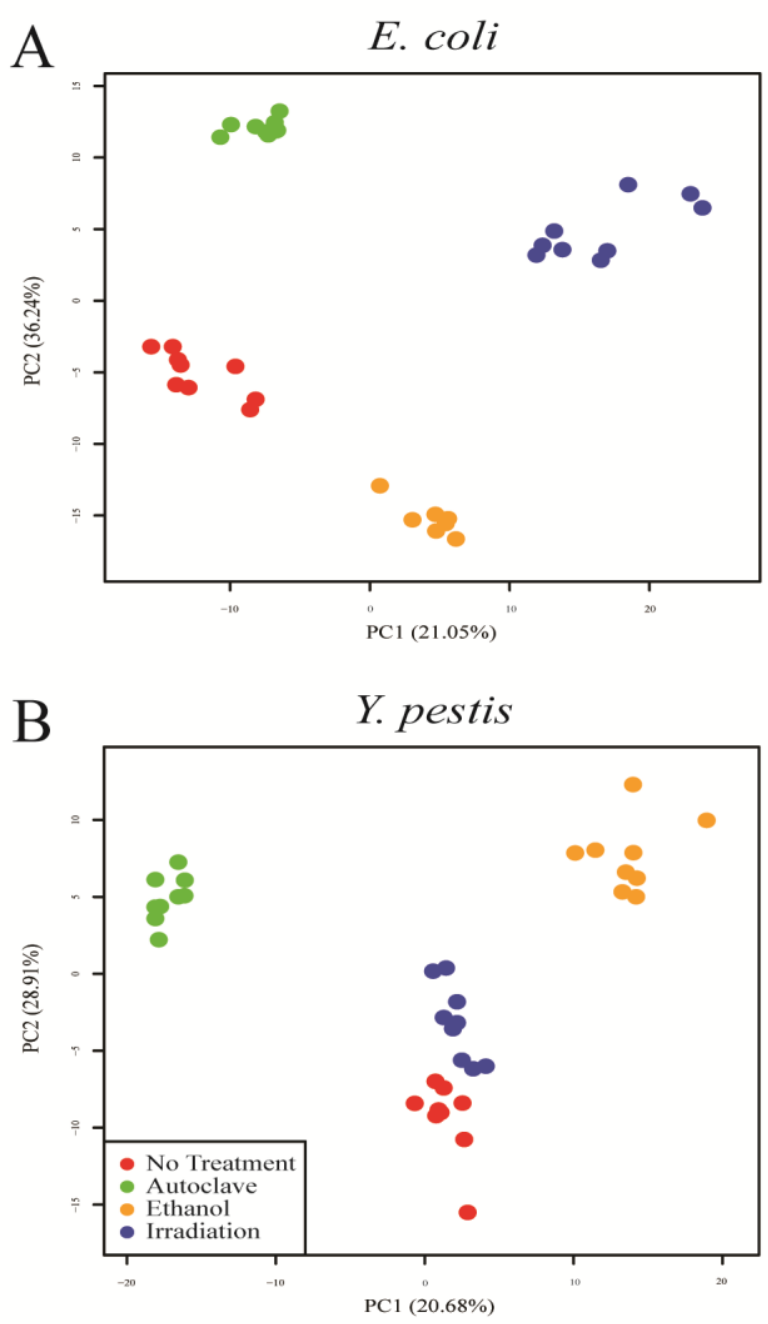

Figure 3: A principal component analysis of the protein abundances generated the plot that represents similarity and differences in protein abundances between each LC-MS/MS run. Samples inactivated by the same method cluster together and do not cluster with samples that underwent different inactivation methods. Each technical replicate is separated for this analysis.

\section{3.3.3 Changes in Observed Abundances of Classes of Proteins:}

Even though protein abundances are relatively static following sample inactivation,

298 significant changes in abundances do occur; some due to random chance. We sought to

299 determine whether proteins that significantly changed in abundance shared any general

300 characteristics that may be attributed to inactivation. To this end, one-sided Fischer exact tests ( $\alpha$

$301<0.05)$ determined that the number of significantly changing proteins localized to particular 
A

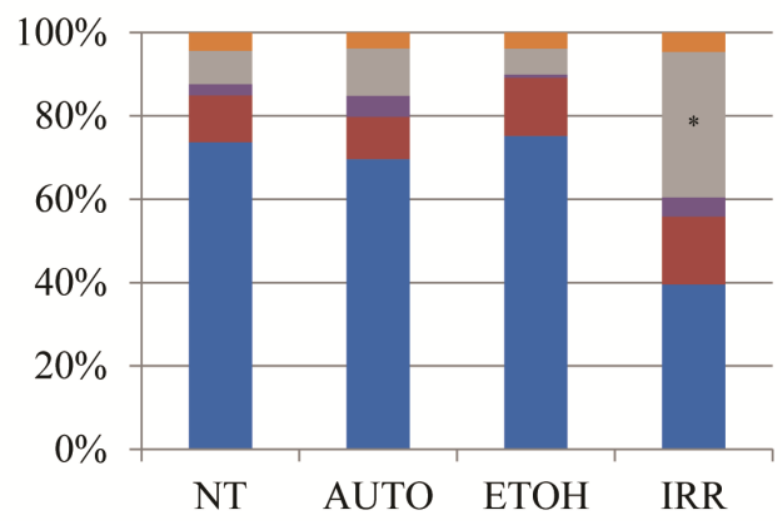

Unknown
B

Y. pestis

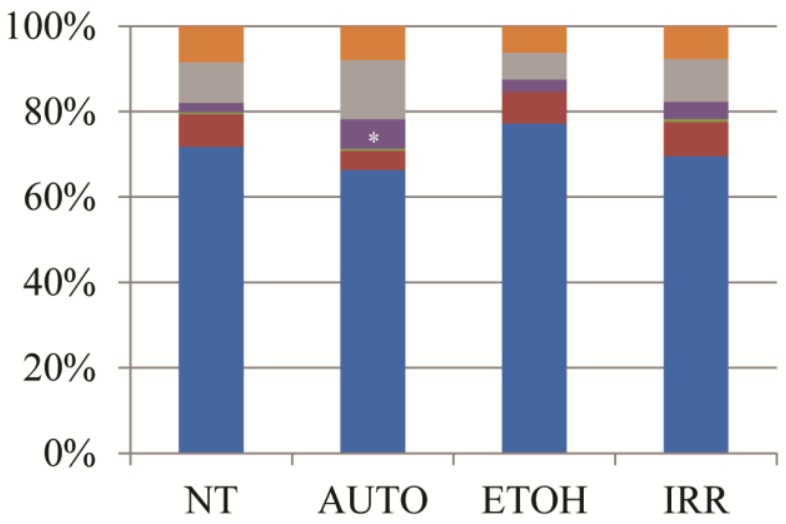

Extracellular

\section{Cytoplasmic}

Membrane

\begin{abstract}
Figure 4: Bar graph showing the subcellular localization of proteins identified in a certain condition. NT (no treatment) consists of all proteins identified. AUTO (autoclave), ETOH (ethanol), and IRR (irradiation) consist of proteins that significantly change in abundance after inactivation in E. coli (A) and Y. pestis (B). Proportions that are significantly larger in the treated samples when compared to the non-treated samples are marked with an asterisk $\left(^{*}\right)$. For this analysis, all data from technical and biological replicates are combined.
\end{abstract}

303 in that particular cellular compartment in the control samples (Figure 4). The background

304 distribution consists of all proteins identified while the test distribution consists of the number of

305 significantly changing proteins after a particular inactivation method, regardless of whether the 306 protein increased or decreased in abundance. For $Y$. pestis, out of all proteins that significantly

307 changed in abundance after autoclaving, the proportion of outer membrane proteins was 308 significantly larger than the proportion of outer membrane proteins in all the proteins identified 309 in the control sample (Figure 4B, Table S1). On the other hand for E. coli, periplasmic proteins 310 exhibited the same behavior after irradiation treatment (Figure 4A, Table S1). This suggests that 311 these proteins were affected more by the inactivation treatment than were proteins localized to 312 other regions of the cell.

\title{
$313 \quad$ 3.3.4 Significant Inactivation Treatment Trends
}




\begin{tabular}{|c|c|c|c|c|c|c|c|c|c|}
\hline & & \multirow[b]{2}{*}{ Subcellular Localization } & \multicolumn{3}{|c|}{ Significantly Changing (localized) } & \multicolumn{3}{|c|}{ All Identified Proteins } & \multirow[b]{2}{*}{$\mathrm{P}$-value } \\
\hline & & & \# increase & \# decrease & Total \# proteins & $\begin{array}{c}\text { \# significant } \\
\text { increase }\end{array}$ & $\begin{array}{c}\text { \# significant } \\
\text { decrease }\end{array}$ & Total \# proteins & \\
\hline \multirow{4}{*}{ E. coli } & \multirow{2}{*}{ AUTO } & Outer Membrane & 6 & 2 & 20 & 43 & 115 & 565 & 0.01091 \\
\hline & & Periplasmic & 0 & 18 & 44 & 43 & 115 & 565 & 0.01713 \\
\hline & ETOH & Cytoplasmic Membrane & 4 & 14 & 62 & 58 & 71 & 593 & 0.04064 \\
\hline & IRR & Periplasmic & 0 & 15 & 44 & 14 & 29 & 576 & $9.43 \mathrm{E}-07$ \\
\hline \multirow{5}{*}{ Y. pestis } & \multirow{2}{*}{ AUTO } & Outer Membrane & 13 & 0 & 24 & 103 & 99 & 580 & 0.002931 \\
\hline & & Periplasmic & 5 & 22 & 47 & 103 & 99 & 580 & 0.000497 \\
\hline & ETOH & Periplasmic & 2 & 13 & 47 & 168 & 73 & 607 & 0.01467 \\
\hline & \multirow{2}{*}{ IRR } & Periplasmic & 0 & 28 & 49 & 187 & 112 & 636 & $1.21 \mathrm{E}-05$ \\
\hline & & Outer Membrane & 13 & 0 & 18 & 187 & 112 & 636 & 0.01539 \\
\hline
\end{tabular}

Table 2: Table detailing the results of one-sided Fischer exact tests for changes in observed abundances of classes of proteins. The test proportion is the ratio of the light grey cells. The background proportion is the ratio of the dark grey cells with the resulting p-value in the following column. Abbreviations used are autoclave (AUTO), ethanol (ETOH), and irradiation (IRR). Data from all biological and technical replicates are combined.

The previous section describes changes in abundance of proteins localized to particular regions of the cell regardless of whether these proteins significantly increased or decreased in abundance. We also explored if the abundances of certain classes of subcellular proteins predominantly changed in the same direction (either significantly increased or decreased) in inactivated samples. These classes of proteins were compared to the overall number of proteins that significantly changed in the same particular direction after inactivation. One-sided Fischer exact tests $(\alpha<0.05)$ were used to identify these trends (Table 2$)$. Some trends occurred in both $Y$. pestis and E. coli. For example, outer membrane proteins generally significantly increased in observed abundance after autoclaving and periplasmic proteins mostly decreased in observed abundance after both autoclaving and irradiation (Table 2). On the other hand, some trends were species specific. In $E$. coli, cytoplasmic membrane proteins generally decreased in observed abundance after ethanol treatment (Table 2). Trends in Y. pestis samples include periplasmic proteins generally decreasing in observed abundance after ethanol treatment and outer membrane proteins largely increasing in observed abundance in the irradiated samples (Table 2).

The majority of observed chaperone and protein folding proteins in both E. coli and $Y$. pestis significantly decreased in abundance after autoclave inactivation. Eleven proteins 
significantly changed in E. coli (9 decreased and 2 increased). Only GroEL and ClpB had significant increases in their observed abundances following autoclaving. In $Y$. pestis, 10 proteins significantly decreased and three significantly increased in observed abundance after autoclave inactivation.

\section{Discussion}

Our results show that inactivating samples by irradiation, autoclaving, and ethanol treatment caused minor changes in the identified proteomes of E. coli and Y. pestis. Metrics such as the distribution of filtered and unfiltered raw scores as well as the density plots of PSMs per protein show few changes in spectral quality as a result of inactivation. In addition, the number of unique peptides and proteins identified between a treated sample and the control demonstrate consistency in the type of peptides and proteins identified. While this and other quality scores for mass spectra showed little variation between inactivated and control samples, PCA plots and Pearson correlation coefficient matrices show that there are systematic differences in observed protein abundances (Figure 2, Figure 3). Even though these differences in abundances are minor, there are discernible trends in the detected abundances of membrane, periplasmic, and protein folding proteins. As an example, significant increases in the observed abundances of outer membrane proteins were found in samples that were autoclaved. These changes in the detected abundances of membrane proteins are presumably related to the morphological changes in the membranes of inactivated cells as seen in Figure 1, perhaps by making many of the proteins more readily extractable and thereby increasing their abundance in inactivated samples.

In contrast to the trend described above, antigen 43, a surface adhesin (Klemm et al., 2004) significantly decreased in observed abundance after autoclave inactivation of E. coli. This protein has an alpha chain on the cell surface and is attached to the outer membrane through the 
beta chain, which is an integral outer membrane protein (Klemm et al., 2004). Previous results demonstrated the alpha subunit releases from the cell surface when heated to $60^{\circ} \mathrm{C}$ (Henderson and Owen, 1999; Klemm et al., 2004), which may help account for the observation of decreased antigen 43 identification within the inactivation condition. To test this hypothesis, each confidently identified peptide was mapped to the sequence of antigen 43. In the control samples, 78 peptides mapping to antigen 43 were identified; 45 of them mapped to the alpha chain while 33 mapped to the beta chain. In the autoclaved samples, only 22 identified peptides could be mapped to antigen 43 , and all of them mapped to the beta chain. This data supports the hypothesis that the alpha chain of the protein was released following autoclaving, and potentially explains why antigen 43 does not follow the trend of $E$. coli outer membrane proteins significantly increasing after autoclave inactivation.

The most distinct trend discovered is that periplasmic proteins significantly decreased in apparent abundance upon autoclave and irradiation inactivation in both Y. pestis and E. coli. Presumably, periplasmic proteins significantly decreased in abundance after being autoclaved since the integrity of the outer membrane is compromised by heat, causing a "leaky" outer membrane (Katsui et al., 1982; Tsuchido et al., 1985). Loss of membrane integrity is supported by the TEM micrographs which distinctly show disrupted outer membranes in the autoclaved and irradiated samples. The disruption of the outer membrane allows periplasmic proteins to leak out of the cell and thus be lost when the sample is pelleted after inactivation and prior to protein digestion.

A similar phenomenon likely occurs with irradiation treatment. Gamma irradiation randomly ionizes molecules; in aqueous culture medium containing cells, the principal result of such irradiation is the creation of oxygen radicals (Gaber, 2005; Kempner, 2001). These oxygen 
radicals react with nearby molecules, resulting in reactions such as lipid peroxidation and consequent degradation and disruption of the lipid membrane (Berroud et al., 1996; Cabiscol et al., 2010). In thymocytes and human red blood cells, irradiation leads to increased permeability of the plasma membrane (Benderitter et al., 2003; K. P. Mishra and B. N. Pandey, 2003). Therefore we suggest that irradiation may also cause permeability of the outer membrane, allowing periplasmic proteins to leak out of the cell. This notion is somewhat supported by the TEM micrographs in which it appears that the cell membrane is disrupted in the irradiated sample, although not to the extent seen in the autoclaved sample.

Finally, among the proteins for which abundances changed significantly in autoclaved samples were several chaperones and other proteins involved in protein folding. Most of these protein folding proteins decreased in abundance following sample inactivation by autoclaving. Since some chaperones localize to the periplasm, subcellular localization could be a confounding factor. However this is unlikely since only two and four of these proteins localized to the periplasm in E. coli and $Y$. pestis, respectively. The reason behind these decreases in thus unclear. Generally, chaperones bind with their denatured client proteins and slowly release them to prevent aggregation of denatured proteins (Hartl and Hayer-Hartl, 2002). We hypothesize that the extreme conditions in an autoclave might cause a cascading affect where the chaperones are unable to keep up with the denaturing environment, causing the chaperones and client proteins to aggregate and precipitate.

The model discussed above leads to an alternative hypothesis regarding the loss of periplasmic proteins in E. coli and $Y$. pestis. Not every periplasmic protein significantly changes in abundance following inactivation, implying that autoclave and irradiation inactivation affect the abundances of only a subset of periplasmic proteins. This observation could be explained if a 
subset of periplasmic proteins is aggregating with chaperones and precipitating. HdeA and HdeB are acidic periplasmic chaperones (Hong et al., 2005, 2012; Kern et al., 2007; Malki et al., 2008) that significantly decrease in observed abundance after autoclaving. They help to resolubilize and renature protein aggregates when the cell $\mathrm{pH}$ returns to neutral (Hong et al., 2012; Kern et al., 2007; Malki et al., 2008). Seventeen client proteins of HdeA (Table 1 in Zhang et al.) were identified in our E. coli data sets. Interestingly 5 of the 17 were found to be significantly decreased in abundance after irradiation inactivation: MalE, OppA, RbsB, DppA, and DegP (Zhang et al., 2011). On the other hand Lpp significantly increased. This could imply that HdeA and HdeB and their client proteins are potentially lost by the same mechanism.

In summary, we have shown that proteomic data quality is unaffected by the three inactivation treatments and, further, that there is little effect on the overall observed bacterial proteome resulting from inactivation of $Y$. pestis and E. coli. However, there are measurable differences in observed protein abundances between inactivated and non-treated cells. These observations suggest that for proteomic experiments where inactivation is required for safety reasons, minimal impact can be expected compared to an untreated system. More specifically, our results demonstrate that ethanol treatment should be given primary preference and irradiation treatment secondary preference to reduce any impact on the observed proteome.

\section{Conclusion}

Since microbiological samples must often be inactivated prior to analysis, particularly if the organisms require BSL-2 or BSL-3 containment, it is important to understand how various inactivation treatments might affect information obtained from an analysis. Our study assessed the impact of ethanol treatment, irradiation, and autoclaving on proteomic data from $Y$. pestis and E. coli. These data showed that, though some differences were detected, the observed protein 
content of cells remained largely unchanged after all three inactivation procedures. Importantly, assessing the different conditions based upon four different quality metrics demonstrated that

While this work provides insight into the effects of three different inactivation methods on two Enterobacteriaceae; it would be advisable to conduct similar studies in other organisms, particularly those whose cell structure is significantly different from our study organisms, such as Gram-positive cells, spores, and Archaea.

\section{Acknowledgements:}

Funding for this work was provided to Pacific Northwest National Laboratory by the Defense Threat Reduction Agency Basic Research Program under DTRA10027IA. We thank Aaron C. Robinson and Heather Engelmann for preliminary work. We also thank Alice Dohnalkova for her help with TEM analysis. A portion of the research was performed using the W.R. Wiley Environmental Molecular Science Laboratory (EMSL), a Department of Energy Office of Science User Facility sponsored by the Office Biological and Environmental Research and located at Pacific Northwest National Laboratory. Pacific Northwest National Laboratory is operated by Battelle Memorial Institute for the United States Department of Energy under contract DE-AC06-76RLO.

\section{References:}

Benderitter, M., Vincent-Genod, L., Pouget, J.P., and Voisin, P. (2003). The cell membrane as a biosensor of oxidative stress induced by radiation exposure: a multiparameter investigation. Radiat. Res. 159, 471-483.

Benjamini, Y., and Hochberg, Y. (1995). Controlling the False Discovery Rate: a Practical and Powerful Approach to Multiple Test. J. R. Stat. Soc. 57, 289-300.

Berroud, A., Le Roy, A., and Voisin, P. (1996). Membrane oxidative damage induced by ionizing radiation detected by fluorescence polarization. Radiat. Environ. Biophys. 35, 289-295. 
Cabiscol, E., Tamarit, J., and Ros, J. (2010). Oxidative stress in bacteria and protein damage by reactive oxygen species. Int. Microbiol. 3, 3-8.

Centers for Disease Control and Prevention (2009). Biosafety in Microbiological and Biomedical Laboratories (BMBL) 5th Edition.

Consortium, T.U. (2014). Activities at the Universal Protein Resource (UniProt). Nucleic Acids Res. 42, D191-D198.

Coohill, T.P., and Sagripanti, J.-L. (2008). Overview of the Inactivation by $254 \mathrm{~nm}$ Ultraviolet Radiation of Bacteria with Particular Relevance to Biodefense. Photochem. Photobiol. 84, 10841090 .

Elias, J.E., and Gygi, S.P. (2007). Target-decoy search strategy for increased confidence in largescale protein identifications by mass spectrometry. Nat. Methods 4, 207-214.

Fenyö, D., and Beavis, R.C. (2003). A method for assessing the statistical significance of mass spectrometry-based protein identifications using general scoring schemes. Anal. Chem. 75, 768774.

Gaber, M.H. (2005). Effect of gamma-irradiation on the molecular properties of bovine serum albumin. J. Biosci. Bioeng. 100, 203-206.

Government Accountability Office (2008). United States Postal Service: Information on the Irradiation of Federal Mail in the Washington, D.C., Area.

Hartl, F.U., and Hayer-Hartl, M. (2002). Molecular Chaperones in the Cytosol: from Nascent Chain to Folded Protein. Science 295, 1852-1858.

Henderson, I.R., and Owen, P. (1999). The Major Phase-Variable Outer Membrane Protein ofEscherichia coli Structurally Resembles the Immunoglobulin A1 Protease Class of Exported Protein and Is Regulated by a Novel Mechanism Involving Dam and OxyR. J. Bacteriol. 181, 2132-2141.

Hong, W., Jiao, W., Hu, J., Zhang, J., Liu, C., Fu, X., Shen, D., Xia, B., and Chang, Z. (2005). Periplasmic Protein HdeA Exhibits Chaperone-like Activity Exclusively within Stomach pH Range by Transforming into Disordered Conformation. J. Biol. Chem. 280, 27029-27034.

Hong, W., Wu, Y.E., Fu, X., and Chang, Z. (2012). Chaperone-dependent mechanisms for acid resistance in enteric bacteria. Trends Microbiol. 20, 328-335.

Huang, D.W., Sherman, B.T., and Lempicki, R.A. (2008). Systematic and integrative analysis of large gene lists using DAVID bioinformatics resources. Nat. Protoc. 4, 44-57.

Huang, D.W., Sherman, B.T., and Lempicki, R.A. (2009). Bioinformatics enrichment tools: paths toward the comprehensive functional analysis of large gene lists. Nucleic Acids Res. 37, 113. 
Katsui, N., Tsuchido, T., Hiramatsu, R., Fujikawa, S., Takano, M., and Shibasaki, I. (1982). Heat-induced blebbing and vesiculation of the outer membrane of Escherichia coli. J. Bacteriol. $151,1523-1531$.

Kelly, R.T., Page, J.S., Luo, Q., Moore, R.J., Orton, D.J., Tang, K., and Smith, R.D. (2006). Chemically Etched Open Tubular and Monolithic Emitters for Nanoelectrospray Ionization Mass Spectrometry. Anal. Chem. 78, 7796-7801.

Kempner, E.S. (2001). Effects of high-energy electrons and gamma rays directly on protein molecules. J. Pharm. Sci. 90, 1637-1646.

Kern, R., Malki, A., Abdallah, J., Tagourti, J., and Richarme, G. (2007). Escherichia coli HdeB Is an Acid Stress Chaperone. J. Bacteriol. 189, 603-610.

Kim, S., Gupta, N., and Pevzner, P.A. (2008). Spectral Probabilities and Generating Functions of Tandem Mass Spectra: a Strike Against Decoy Databases. J. Proteome Res. 7, 3354-3363.

Klemm, P., Hjerrild, L., Gjermansen, M., and Schembri, M.A. (2004). Structure-function analysis of the self-recognizing Antigen 43 autotransporter protein from Escherichia coli. Mol. Microbiol. 51, 283-296.

K. P. Mishra, and B. N. Pandey (2003). Oxidative membrane damage and its involvement in gamma radiation-induced apoptotic cell death. Int. J. Radiat. Res. 1, 17-22.

Lasch, P., Nattermann, H., Erhard, M., Stämmler, M., Grunow, R., Bannert, N., Appel, B., and Naumann, D. (2008). MALDI-TOF Mass Spectrometry Compatible Inactivation Method for Highly Pathogenic Microbial Cells and Spores. Anal. Chem. 80, 2026-2034.

Li, M., Gray, W., Zhang, H., Chung, C.H., Billheimer, D., Yarbrough, W.G., Liebler, D.C., Shyr, Y., and Slebos, R.J.C. (2010). Comparative Shotgun Proteomics Using Spectral Count Data and Quasi-Likelihood Modeling. J. Proteome Res. 9, 4295-4305.

Liu, H., Sadygov, R.G., and Yates, J.R. (2004). A Model for Random Sampling and Estimation of Relative Protein Abundance in Shotgun Proteomics. Anal. Chem. 76, 4193-4201.

Malki, A., Le, H.-T., Milles, S., Kern, R., Caldas, T., Abdallah, J., and Richarme, G. (2008). Solubilization of Protein Aggregates by the Acid Stress Chaperones HdeA and HdeB. J. Biol. Chem. 283, 13679-13687.

509 Mayampurath, A.M., Jaitly, N., Purvine, S.O., Monroe, M.E., Auberry, K.J., Adkins, J.N., and 510 Smith, R.D. (2008). DeconMSn: a software tool for accurate parent ion monoisotopic mass 511 determination for tandem mass spectra. Bioinforma. Oxf. Engl. 24, 1021-1023.

512 Petyuk, V.A., Mayampurath, A.M., Monroe, M.E., Polpitiya, A.D., Purvine, S.O., Anderson, 513 G.A., Camp, D.G., 2nd, and Smith, R.D. (2010). DtaRefinery, a software tool for elimination of 514 systematic errors from parent ion mass measurements in tandem mass spectra data sets. Mol. 515 Cell. Proteomics MCP 9, 486-496. 
Rose, L.J., and O'Connell, H. (2009). UV Light Inactivation of Bacterial Biothreat Agents. Appl.

520 Microbiol. 77, 7289-7295.

521 Talbot, S.R., Russmann, H., Köhne, S., Niederwöhrmeier, B., Grote, G., and Scheper, T. (2010).

522 Effects of inactivation methods on the analysis of Bacillus atrophaeus endospores using real-time 523 PCR and MALDI-TOF-MS. Eng. Life Sci. 10, 109-120.

524 Tsuchido, T., Katsui, N., Takeuchi, A., Takano, M., and Shibasaki, I. (1985). Destruction of the 525 outer membrane permeability barrier of Escherichia coli by heat treatment. Appl. Environ.

526 Microbiol. 50, 298-303.

527 Une, T., and Brubaker, R.R. (1984). In vivo comparison of avirulent Vwa- and Pgm- or Pstr 528 phenotypes of yersiniae. Infect. Immun. 43, 895-900.

529 Vatansever, F., Ferraresi, C., de Sousa, M.V.P., Yin, R., Rineh, A., Sharma, S.K., and Hamblin, 530 M.R. (2013). Can biowarfare agents be defeated with light? Virulence 4, 796-825.

531 Waje, C.K., Jun, S.Y., Lee, Y.K., Kim, B.N., Han, D.H., Jo, C., and Kwon, J.H. (2009).

532 Microbial quality assessment and pathogen inactivation by electron beam and gamma irradiation 533 of commercial seed sprouts. Food Control 20, 200-204.

534 Wattam, A.R., Abraham, D., Dalay, O., Disz, T.L., Driscoll, T., Gabbard, J.L., Gillespie, J.J., 535 Gough, R., Hix, D., Kenyon, R., et al. (2014). PATRIC, the bacterial bioinformatics database and 536 analysis resource. Nucleic Acids Res. 42, D581-D591.

Whitney, E.A.S., Beatty, M.E., Taylor, T.H., Weyant, R., Sobel, J., Arduino, M.J., and Ashford, D.A. (2003). Inactivation of Bacillus anthracis Spores. Emerg. Infect. Dis. 9, 623-627.

Yu, N.Y., Wagner, J.R., Laird, M.R., Melli, G., Rey, S., Lo, R., Dao, P., Sahinalp, S.C., Ester, M., Foster, L.J., et al. (2010). PSORTb 3.0: improved protein subcellular localization prediction with refined localization subcategories and predictive capabilities for all prokaryotes. Bioinformatics 26, 1608-1615.

Zaki, A.N. (2010). Biosafety and biosecurity measures: management of biosafety level 3 facilities. Int. J. Antimicrob. Agents 36, Supplement 1, S70-S74.

Zhang, M., Lin, S., Song, X., Liu, J., Fu, Y., Ge, X., Fu, X., Chang, Z., and Chen, P.R. (2011). A genetically incorporated crosslinker reveals chaperone cooperation in acid resistance. Nat. Chem. Biol. 7, 671-677. 

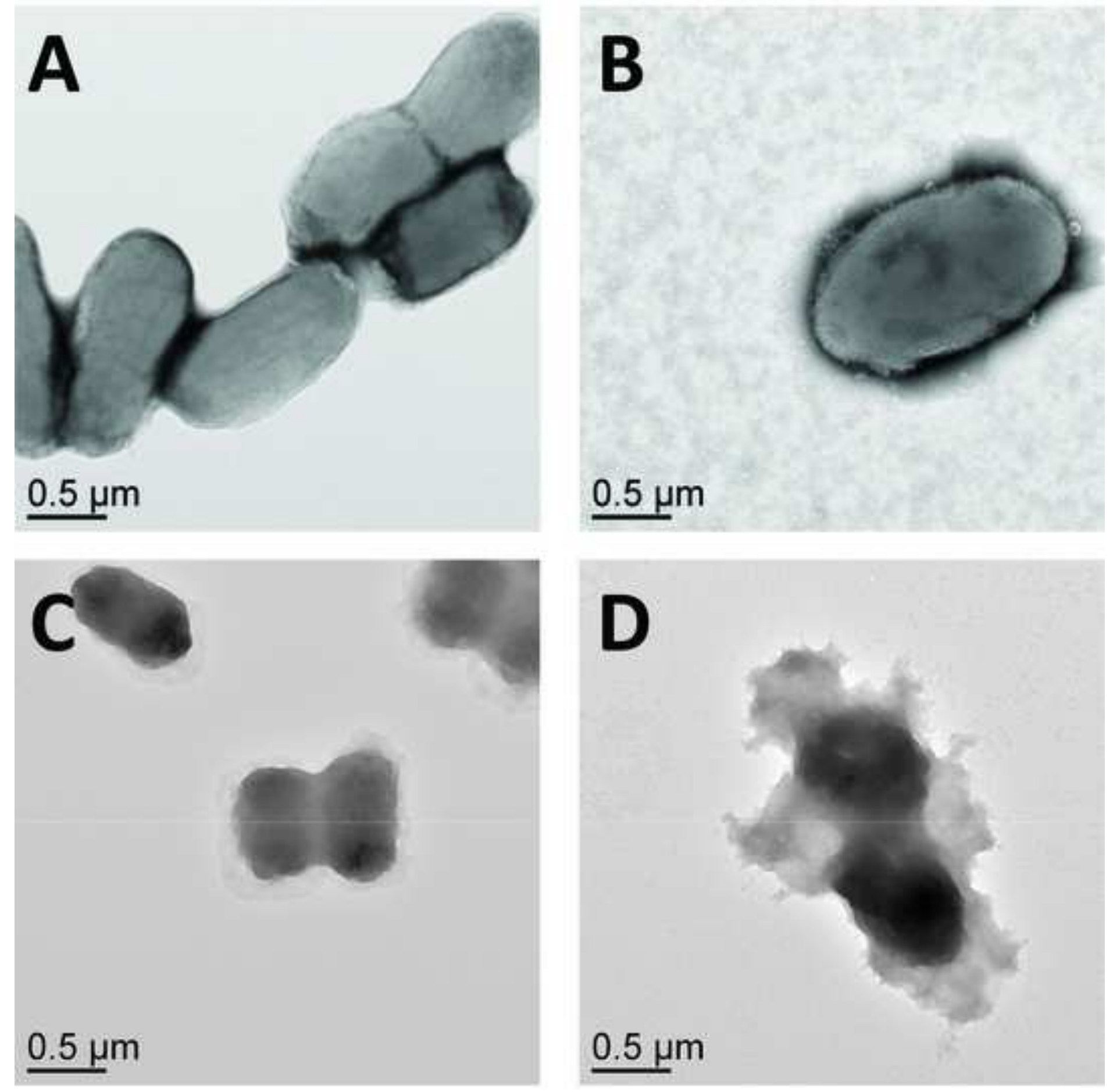


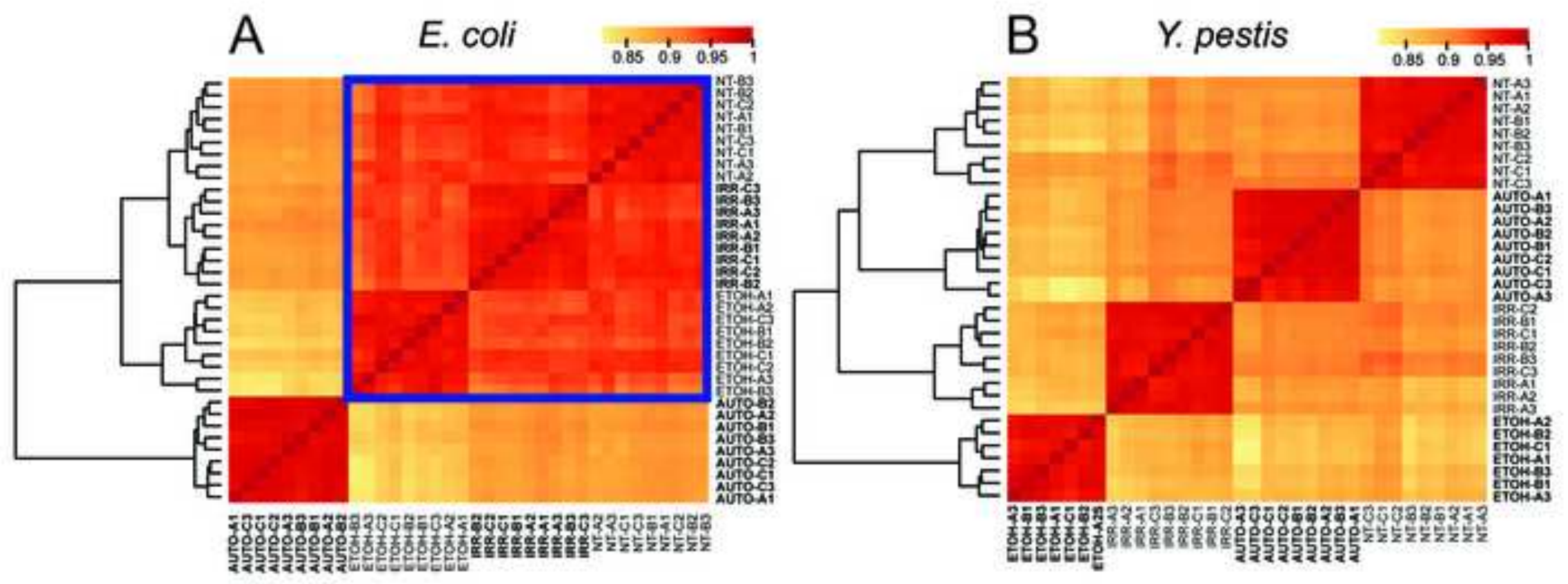



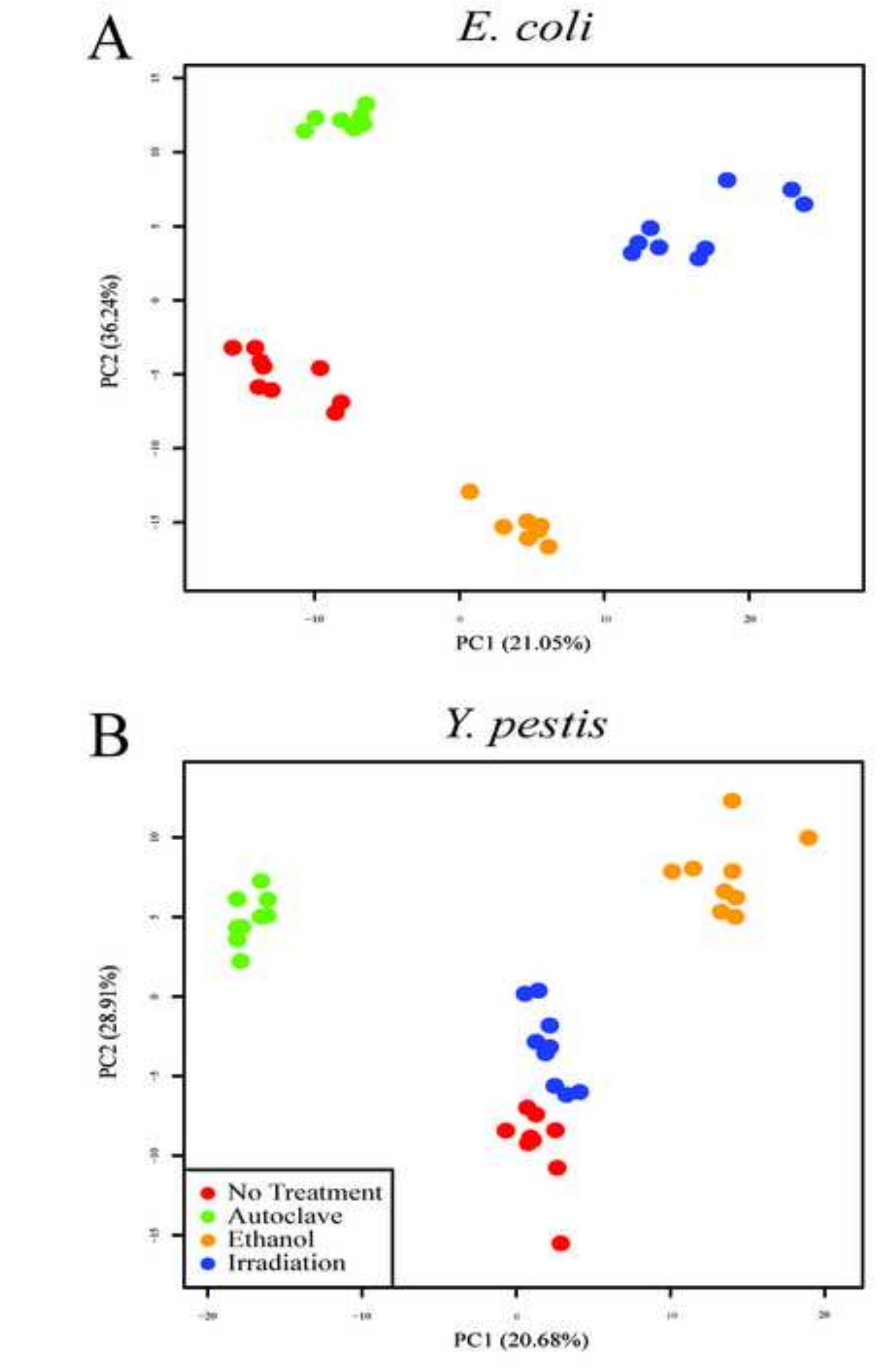

Figure 3

Figure 3

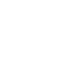

(

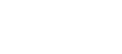


A

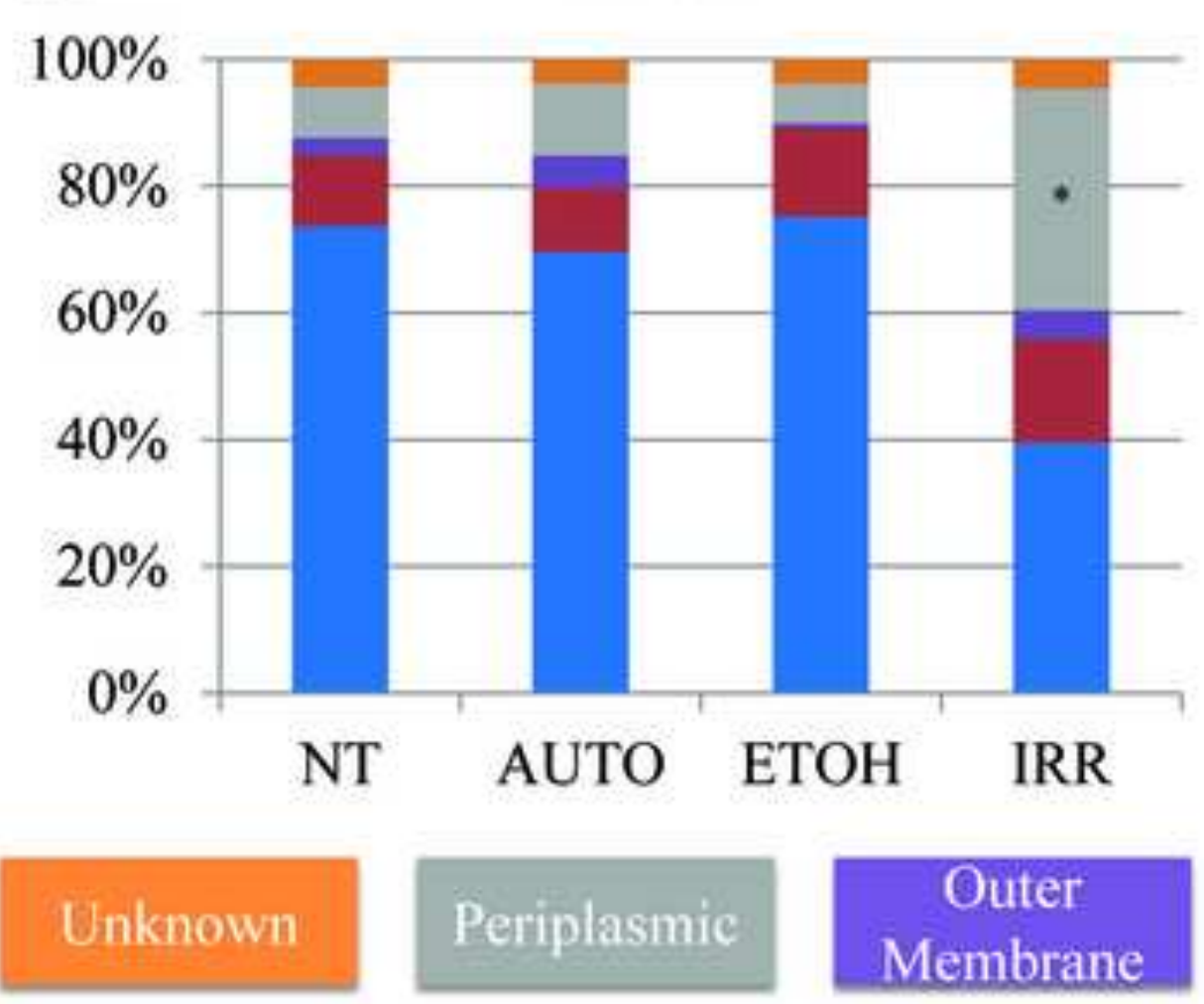

B

Y. pestis

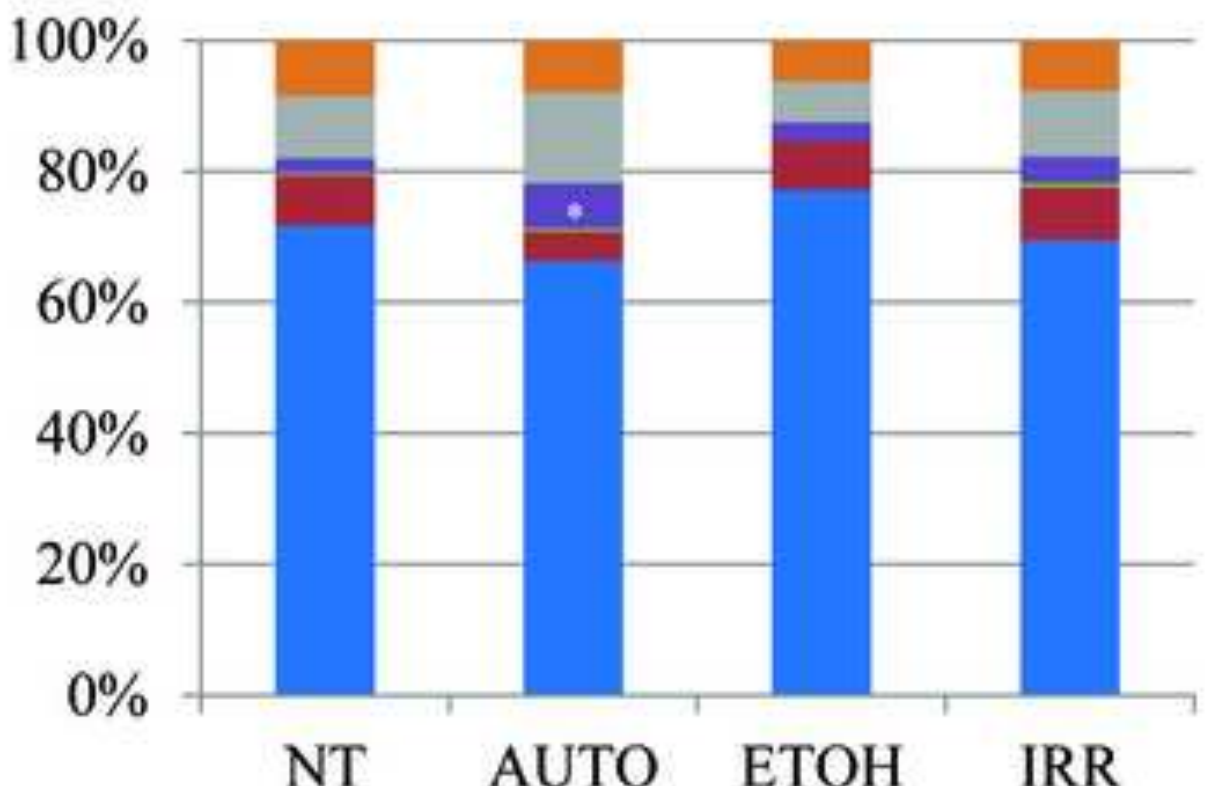

Extracellular

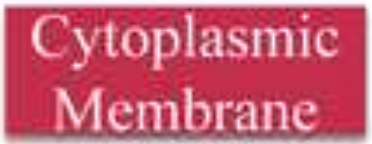

